\title{
Der Wahlschweizer schätzt den kollegialen Umgangston
}

\section{Nathalie Zeindler}

Freie Journalistin

Rund ein Drittel der Ärztinnen und Ärzte in der Schweiz kommt aus dem Ausland. Weshalb haben sie sich für ein Leben hierzulande entschieden? Welches sind kulturelle Stolpersteine, die es zu umschiffen gilt? Und was halten sie von der Schweizer Gesundheitspolitik? Diesen und weiteren Fragen möchten wir in der in loser Folge erscheinenden Artikelserie «Grüezi Schweiz» nachgehen. In dieser Ausgabe stellen wir Dr. Frank Sachers vor, der hierzulande die Augenheilkunde für sich entdeckt hat.

In den 80er Jahren sahen sich Medizinstudentinnen und -studenten in Deutschland mit düsteren beruflichen Zukunftsprognosen konfrontiert. Die Arbeitslosigkeit stieg stetig an, da die Zahl der Bewerberinnen und Bewerber diejenige der offenen Stellen deutlich überstieg. In jener Zeit tauchte der Begriff Ärzteschwemme auf.

Auch der heute 56-jährige Frank Sachers, damals Studierender an der Medizinischen Fakultät der Universität Ulm, gehörte zu den Betroffenen: «Die Folge dieser Entwicklung war die Einführung der

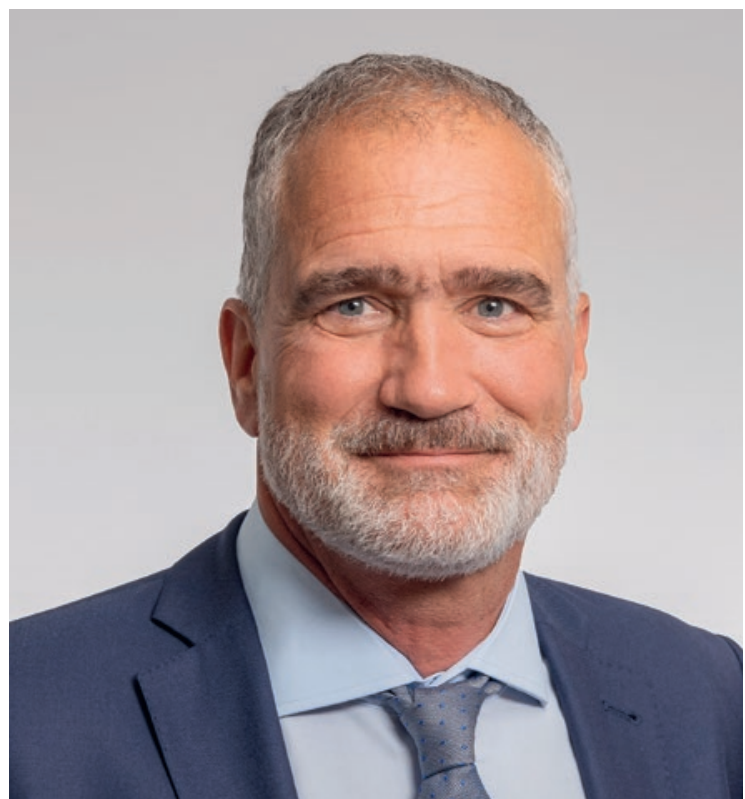

Dr. Frank Sachers leitet seit 2007 das Augenzentrum Bahnhof Basel. 18-monatigen Phase des Arztes im Praktikum (AIP) nach Abschluss des Studiums mit einer deutlich tieferen Entlöhnung im Vergleich zum Assistenzarzt», sagt er. Auch in der Schweiz würden besagte Fachkräfte

Der Mediziner schätzte die im Gegensatz zu Deutschland weniger spürbaren Hierarchiestufen sowie den kollegialen Umgangston.

deutlich schlechter bezahlt als Assistenten, doch seien zumindest genügend interessante Stellenangebote vorhanden.

Da der in Stuttgart geborene Mediziner das Kantonsspital Aarau aus seiner Zeit als Unterassistent in der chirurgischen Abteilung bereits kannte und die im Gegensatz zu Deutschland weniger spürbaren Hierarchiestufen sowie den kollegialen Umgangston schätzte, sah er davon ab, sich andernorts zu bewerben - nicht zuletzt auch der Liebe wegen. Man dürfe zudem nicht vergessen, dass ausländische Medizinerinnen und Mediziner vor 30 Jahren in der Schweiz eher als Exoten galten. Die Personenfreizügigkeit lag damals noch in weiter Ferne.

\section{Faszination Augenheilkunde}

Als Arzt im Praktikum landete Frank Sachers in der Augenheilkunde des Kantonsspitals Aarau, und schon nach kurzer Zeit wurde der Posten in eine Assistenzarztstelle umgewandelt. «Anfänglich war ich der Meinung, dass die Augenheilkunde lediglich aus geröteten Augen und Druckmessungen besteht. Doch es handelt 


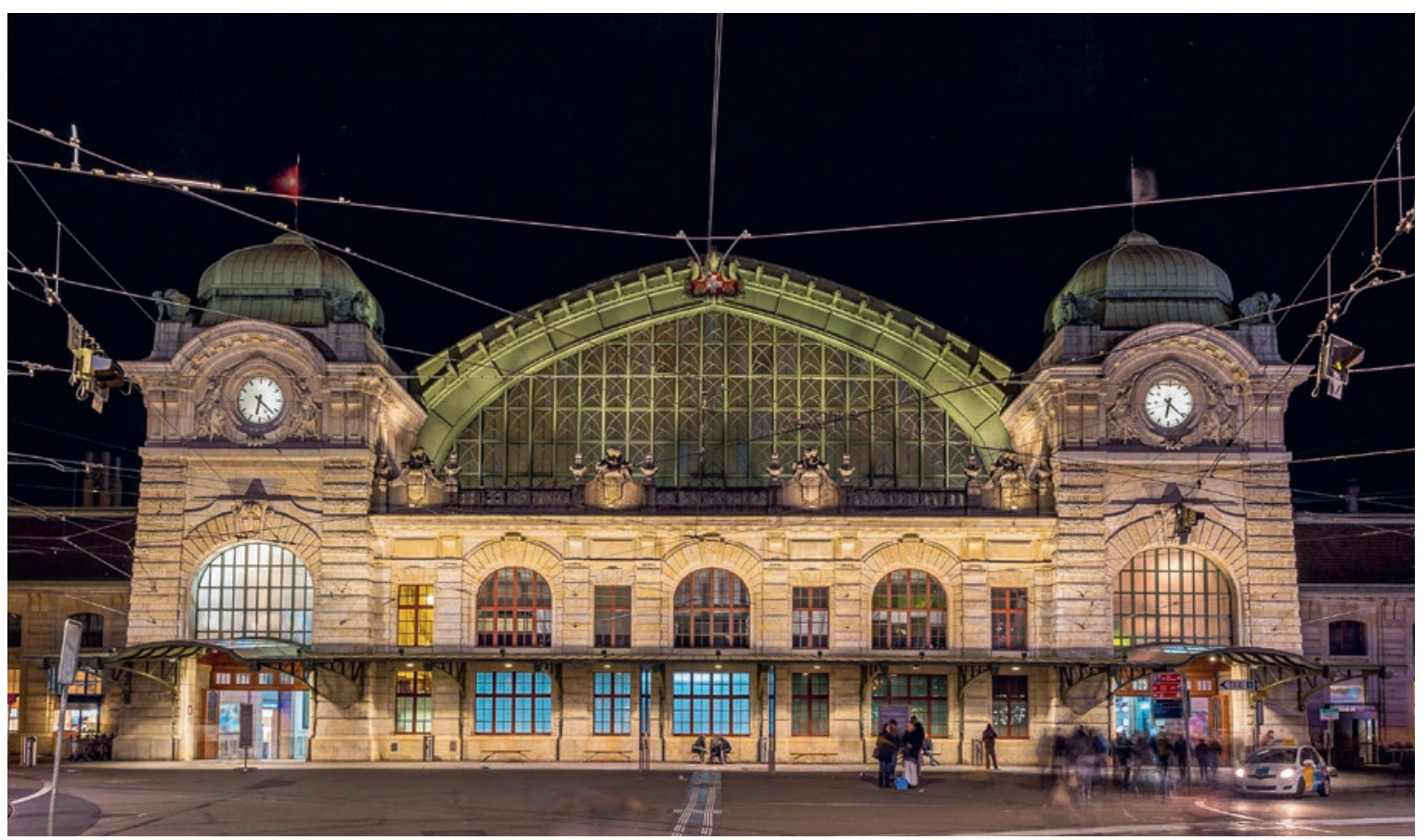

In unmittelbarer Nähe zum Bahnhof Basel SBB empfängt Dr. Frank Sachers seine Patienten.

sich um eine sehr vielseitige Disziplin.» In den folgenden Jahren wandte sich Frank Sachers immer mehr der operativen Augenheilkunde zu.

Mittlerweile führt er seit 2007 das Augenzentrum Bahnhof Basel, ein grosses Zentrum für ambulante Ophthalmochirurgie und klassische Ophthalmologie. «In den letzten Jahren ist in meinem Fachgebiet eine gewisse Spaltung zwischen konservativ und operativ tätigen Kolleginnen und Kollegen feststellbar. Die Weiterbildung in Ophthalmochirurgie hat zwar zu einer Qualitätsverbesserung geführt, aber auch die Hürden für eine operative Tätigkeit erhöht», so Dr. Sachers.

\section{Er stellt fest, dass ältere Techniken seltener} angewandt werden, wegen mangelnder Erfahrung schlechtere Resultate liefern und schliesslich aus dem Spektrum verschwinden.

Der Frauenanteil habe in den letzten Jahren zugenommen, und Teilzeitmodelle seien heute eher die Norm als die Ausnahme. Im Bereich der chirurgischen Ausbildung sei das nicht immer einfach, sagt der Facharzt und fügt an: «Eine gute Organisation sowie genügend Flexibilität für alle Beteiligten ermöglichen dennoch eine ausreichende Kontinuität.»

Ein Arbeitspensum von 70 bis 80 Prozent während der operativen Ausbildung hält der Leiter des Augenzentrums Bahnhof Basel, einer Weiterbildungsklinik C, für unabdingbar, um ein hohes technisches Niveau zu erreichen. Er legt Wert auf Kommunikation mit den Patienten und klassische klinische Diagnostik, die heute neben den modernen apparativen Methoden ein Dornröschendasein fristet. Frank Sachers ist deshalb überzeugt, dass nicht nur eine gute Operationstechnik zu einem guten Resultat beiträgt, sondern vor allem auch eine individuelle und an die Situation des Patienten angepasste Indikationsstellung.

\section{Vielseitigkeit ist Trumpf}

Während seiner langen Operationstätigkeit konnte sich Dr. Sachers verschiedene Techniken sowohl in der Vorder- als auch in der Hinterabschnittschirurgie aneignen. Dieser Erfahrungsschatz hilft ihm heute, auch komplexe Ausgangssituationen erfolgreich anzugehen. Er stellt fest, dass ältere Techniken seltener angewandt werden, wegen mangelnder Erfahrung schlechtere Resultate liefern und schliesslich aus dem Spektrum verschwinden. Allerdings wären diese möglicherweise für die eine oder andere Ausgangssituation besser geeignet als modernere Techniken. Neu sei nicht immer besser, sagt er mit Nachdruck.

Nicht nur die Weiterbildung beschäftigt Frank Sachers, sondern auch die Tarifpolitik.

Als langjähriges Mitglied der Tarifkommission der Schweizerischen Ophthalmologischen Gesellschaft ist er stolz darauf, dass Fallpauschalen für die Abrechnung ambulanter Eingriffe, beispielsweise die Kataraktoperation oder intravitreale Injektionen, seit vielen Jahren angewendet werden. Die Ophthalmologie nehme in dieser Hinsicht eine gewisse Vorreiterrolle ein. 
In den vergangenen Jahren kenne die Abgeltung für ambulante Tarife allerdings nur die Richtung nach unten. Inzwischen sei der Tarif für die ganze Operation tiefer als vor 20 Jahren das Operationshonorar des Chirurgen bei stationärer Durchführung des Eingriffs. Auch im Wechsel von stationärer zu ambulanter Chirurgie gelte die Augenheilkunde als Paradebeispiel. Die Chirurgie des Grauen Stars werde inzwischen nahezu immer ambulant durchgeführt, und auch in der Netzhautchirurgie seien dementsprechende Tendenzen sichtbar.

\section{Zwei Länder, zwei Systeme}

Natürlich existieren grundlegende Unterschiede bezüglich der Gesundheitssysteme zwischen Deutschland und der Schweiz, und man hüte sich vor einer Dis-

\section{«Abschalten, entspannen und einmal Zeit für sich selbst haben ist eine wahre Kunst.»}

kussion im Sinne besser-schlechter. Dennoch lohne sich hie und da ein Blick über den Gartenzaun, meint Dr. Sachers. So habe die Schweiz vor einigen Jahren die SwissDRG zu einem Zeitpunkt eingeführt, als man in Deutschland bereits deren Nutzen kritisch zu hinterfragen begann und gar darüber diskutierte, besagtes System wieder abzuschaffen.

«Natürlich frage ich mich manchmal, warum in der Schweiz Anpassungen nur allmählich stattfinden. Die Diskussionen über steigende Krankenkassenprämien begleiten uns seit Jahren", meint der Facharzt. Gleichzeitig komme die notwendige Strukturbereinigung der Spitallandschaft nicht in die Gänge, und der Föderalismus sorge, wie in der Nordwestschweiz deutlich zu sehen sei, auch nicht gerade für eine Beschleunigung des Strukturwandels.

Pro Jahr führt Dr. Sachers über 2500 intraokulare Eingriffe durch. Kein Auge gleiche dem anderen, was auch bedeute, dass jede Operation nach jahrelanger Tätig- keit noch immer als Herausforderung angesehen werden könne - mit dem Ziel, für den Patienten das Optimum herauszuholen. «Trotz der hohen Fallzahlen sind mir der Kontakt zum Patienten und die präoperative Diagnostik sowie die Aufklärung sehr wichtig. Entsprechend kann ich mir ein Leben als 'operierender Technokrat' nicht vorstellen.»

\section{Heimat im Dreiländereck}

Im Dreiländereck hat sich der inzwischen zweifache Familienvater mittlerweile gut eingelebt. «Wenn man wie ich aus dem süddeutschen Raum stammt, fühlt man sich in der Nordwestschweiz wohl. Das hat auch damit zu tun, dass sich die beiden Dialekte nicht wesentlich voneinander unterscheiden.»

Nach Arbeitsschluss schwimmt der Wahlschweizer im Sommer gerne im nahegelegenen Rhein, zudem schätzt er das kulturelle Angebot.

Entspannung findet er auch in den Bergen des Lötschentals, und seit vielen Jahren sucht er auf Safaris in Afrika zusammen mit seiner Frau Abstand zu seinem Beruf: «Mikrochirurgie stellt eine hohe körperliche Belastung dar. Ich stelle fest, dass ich mit 56 Jahren etwas mehr Ruhepausen benötige als früher», sagt er und fügt hinzu: «Abschalten, entspannen und einmal Zeit für sich selbst haben ist eine wahre Kunst.»

\section{Bildnachweis}

Foto Frank Sachers: zVg

Foto Bahnhof Basel: Leonid Andronov | Dreamstime.com

Für unsere Serie «Grüezi Schweiz» suchen wir ausländische Ärztinnen und Ärzte, die uns einen Einblick in ihr Leben und ihren Berufsalltag gewähren. Wir freuen uns über eine Kontaktaufnahme: matthias.scholer[at]emh.ch

Pour notre rubrique «Bonjour la Suisse», nous recherchons des médecins étrangers pour nous raconter ce qu'ils pensent de leur existence et de leur quotidien professionnel. C'est avec plaisir que nous vous lirons: matthias.scholer[at]emh.ch. 